\title{
COMPLETELY SEMISIMPLE SEMIGROUPS AND EPIMORPHISMS
}

\author{
PETER M. HIGGINS
}

\begin{abstract}
It is proved that a completely semisimple semigroup $U$ cannot be properly epimorphically embedded in another semigroup if $U$ has no infinite chain of $J$-classes.
\end{abstract}

Let $U, S$ be semigroups with $U \subseteq S$. Following Howie and Isbell [8] we say $U$ dominates an element $d \in S$ if for every semigroup $T$ and all homomorphisms $f: S \rightarrow T$ and $g: S \rightarrow T, u f=u g$ for all $u \in U$ implies $d f=d g$. The set of all elements of $S$ dominated by $U$ is called the dominion of $U$ in $S$ and is denoted by $\operatorname{Dom}(U, S)$. It is easily verified that $\operatorname{Dom}(U, S)$ is a subsemigroup of $S$ containing $U$. We say $U$ is closed in $S$ if $\operatorname{Dom}(U, S)=U$ and $U$ is absolutely closed if $U$ is closed in every containing semigroup $S$. A semigroup $U$ is saturated if $\operatorname{Dom}(U, S) \neq S$ for every properly containing semigroup $S$.

Let $f: S \rightarrow T$ be a morphism of semigroups. Then $f$ is an epimorphism (epi for short) if for every pair of morphisms $g: T \rightarrow V$ and $h: T \rightarrow V, f g=f h$ implies $g=h$. It is easy to check that a morphism $f: S \rightarrow T$ is epi if and only if the inclusion $i: f(S) \rightarrow T$ is epi, and if $U \subseteq S$ then $\operatorname{Dom}(U, S)=S$ if and only if $i: U \rightarrow S$ is epi, whereupon we say that $U$ is epimorphically embedded in $S$.

Most results concerning semigroup dominions and epimorphisms are based on the following result.

Result 1 (Isbell's Zigzag Theorem [7, Theorem 2.13]). Let $U$ be a subsemigroup of a semigroup $S$ and let $d \in S$. Then $d \in \operatorname{Dom}(U, S)$ if and only if $d \in U$ or there is a series of factorizations of $d$ as follows:

$$
d=u_{0} y_{1}=x_{1} u_{1} y_{1}=x_{1} u_{2} y_{2}=x_{2} u_{3} y_{2}=\cdots=x_{m} u_{2 m-1} y_{m}=x_{m} u_{2 m},
$$

where $m \geq 1, u_{i} \in U, x_{i}, y_{i} \in S$ with $u_{0}=x_{1} u_{1}, u_{2 i-1} y_{i}=u_{2 i} y_{i+1}, x_{i} u_{2 i}=$ $x_{i+1} u_{2 i+1}(1 \leq i \leq m-1), u_{2 m-1} y_{m}=u_{2 m}$.

Such a series of factorizations is called a zigzag in $S$ over $U$ with value d, length $m$ and spine $u_{0}, u_{1}, \ldots, u_{2 m}$.

Semigroup dominions can also be expressed in terms of special semigroup amalgams [7, Theorem 2.3]. In particular $U$ is absolutely closed if and only if every special amalgam with core $U$ is strongly embeddable in a semigroup.

Most notable results in this area have been to the effect that certain classes consist entirely of absolutely closed or of saturated semigroups.

Received by the editors February 10, 1985.

1980 Mathematics Subject Classification. Primary 20M99.

Key words and phrases. Semigroup, epimorphism, completely semisimple. 
For example, it was shown by Howie and Isbell [8] that right simple semigroups, finite monogenic semigroups and inverse semigroups are absolutely closed. Scheiblich and Moore [10] showed that the total transformation semigroup, $\tau_{x}$, is absolutely closed; a result also proved in Shoji $[\mathbf{1 1}]$ and by Hall $[\mathbf{1}]$ whose proof also works for the semigroup of partial transformations on a set.

Howie and Isbell [8] proved that any commutative semigroup that satisfies $M_{J}$, the minimum condition on $J$ classes, is saturated and the same is true of any finite permutative semigroup (a semigroup is permutative if it satisfies a nontrivial permutation identity) [6]. Another result due to the author $[3]$ is that generalized inverse semigroups are saturated (regular semigroups whose idempotents form a normal subband). Finally, a strong result due to Hall and Jones [2] is that every completely semisimple semigroup with a finite number of $J$-classes is saturated; in particular this includes all completely [0-] simple and finite regular semigroups. We prove a stronger result below.

On the other hand the $2 \times 2$ rectangular band is not absolutely closed [8] and the 3-element null semigroup can have an infinite dominion [1]. Furthermore there are semigroups of each of the following types which are not saturated; commutative cancellative semigroups (the injection of the natural numbers into the integers under addition provides an example), subsemigroups of finite inverse semigroups [9], commutative, periodic semigroups $[\mathbf{4}]$, and bands [5]. Indeed recently Trotter $[\mathbf{1 2}]$ has constructed a band with a properly epimorphically embedded subband.

The following theorem was announced at the Marquette Semigroup Conference in 1984. The result will appear in the Proceedings of that conference.

THEOREM 2. A completely semisimple semigroup $U$ is saturated if it has no infinite chain of J-classes.

Before we give the proof we make a couple of technical observations concerning zigzags. Let $Z$ be a zigzag as described in Result 1 . If the length of $Z$ is minimum amongst all those zigzags in $S$ over $U$ with value $d$, then $x_{i}, y_{i} \in S \backslash U$ for all $i=1,2, \ldots, m$. Next suppose that $U$ is properly epimorphically embedded in $S$, and that two successive lines of a zigzag $Z$ of minimum length $m$, with value $d \in S \backslash U$, are $x_{i} u_{2 i} y_{i+1}=x_{i+1} u_{2 i+1} y_{i+1}(1 \leq i \leq m-1)$. Then, since $y_{i+1} \in S \backslash U$ we have, by the Zigzag Theorem, $y_{i+1}=a y_{i+1}^{\prime}$ for some $a \in U, y_{i+1}^{\prime} \in S \backslash U$. We may then construct a modified zigzag $Z^{\prime}$, with value $d$, where the two given lines are replaced by $x_{i} u_{2 i} a y_{i+1}^{\prime}=x_{i+1} u_{2 i+1} a y_{i+1}^{\prime}$ because the necessary equalities are provided by $u_{2 i-1} y_{i}=u_{2 i} a y_{i+1}^{\prime}, x_{i} u_{2 i} a=x_{i+1} u_{2 i+1} a$ and $u_{2 i+1} a y_{i+1}^{\prime}=u_{2 i+2} a y_{i+1}^{\prime}$. A similar remark also applies to the two initial lines of the zigzag: $u_{0} y_{1}=x_{1} u_{1} y_{1}$. We call the process of passing from $Z$ to $Z^{\prime}$ "expansion of $Z$ at $y_{i+1}$ via the factorization $y_{i+1}=a y_{i+1}^{\prime}$ ". Of course we can perform this modification for every $y_{i}$ if we choose. There is a dual comment which applies to the $x_{i}$ which is also true.

It will be understood throughout that Green's relations are relations in $U$.

PROOF OF THEOREM 2. Suppose that $U$ is a completely semisimple semigroup with no infinite chain of $J$-classes properly epimorphically embedded in $S$.

Take $d \in S \backslash U$. Let $C_{d}$ be the collection of all $J$-classes $J$, such that some $u \in J$ is the first spine member of some zigzag of minimum length in $S$ over $U$ with value 
$d$. Denote the collection of all minimal members of $\mathcal{C}_{d}$ by $\bar{C}_{d}$ and put

$$
J_{R}=\bigcup_{d \in S \backslash U} \bar{C}_{d}
$$

The dual collection of $J$-classes will be denoted by $J_{L}$.

Take $J$ to be a maximal member of $J_{R} \cup J_{L}$. Without loss we assume that $J \in J_{R}$, so that there exists $d \in S \backslash U$ such that $d=u_{0} y_{1}$ say, is the first line of the zigzag $Z$ of minimum length $m$ in $S$ over $U$ with value $d, u_{0} \in J$, and if $d=u y$ is the first line of another such zigzag, then $J_{u} \nless J$. The maximality condition on $J$ guarantees that the $J$-class of the first spine member of $Z$ is invariant under any expansion. We may also assume that the $J$-class corresponding to each particular spine member is invariant under expansion at $x_{i}$ or $y_{i}(1 \leq i \leq m)$, because we may expand $Z$ at each $x_{i}$ or $y_{i}$ until the $J$-class of each spine member is fixed under any further expansions, which must occur as $U$ satisfies the descending chain condition on $J$-classes. We shall assume that this process has been carried out.

We show that $u_{0}, u_{1}, \ldots, u_{2 m-1} \in J$. We have $u_{0} \in J$, so assume inductively that $u_{0}, u_{1}, \ldots, u_{2 i} \in J(0 \leq i \leq m-1)$. We first prove that $J \leq J_{u_{2 i+1}}$. First if $i=0$ the equality $u_{0}=x_{1} u_{1}$ implies that $u_{0}=u_{0} u_{1}^{\prime} u_{1}\left(u_{1}^{\prime} \in V\left(u_{1}\right)\right)$, whence $J \leq J_{u_{1}}$. If $i>0$ we factorize $x_{i}$ as $x_{i}^{\prime} a_{i}$, where $a_{i} \in J_{L}$. We obtain

$$
J=J_{u_{2 i}}=J_{a_{i} u_{2 i}} \leq J_{a_{i}},
$$

where the second equality is justified by the invariance of the $J$-class under expansion. However, $J<J_{a_{i}}$ is impossible because of the maximality condition on $J$, and thus we have equality throughout in (1) and $a_{i}, u_{2 i}, a_{i} u_{2 i} \in J$.

Since $R_{a_{i} u_{2 i}} \leq R_{a_{i}}, a_{i} u_{2 i} J a_{i}$ and $U$ is completely semisimple, it follows that $R_{a_{i} u_{2 i}}=R_{a_{i}}$. Hence $a_{i}=a_{i} u_{2 i} t_{i}$ for some $t_{i} \in U^{1}$, whence we have

$$
x_{i}=x_{i}^{\prime} a_{i}=x_{i}^{\prime} a_{i} u_{2 i} t_{i}=x_{i} u_{2 i} t_{i}=x_{i+1} u_{2 i+1} t_{i} .
$$

Consider the zigzag $Z^{\prime}$ which results from expansion of $Z$ at $x_{i}$ via the factorization $x_{i}=x_{i+1} u_{2 i+1} t_{i}$. Since the $J$-class of each spine member of $Z$ is invariant under any expansion we obtain

$$
J=J_{u_{2 i}}=J_{u_{2 i+1} t_{i} u_{2 i}} \leq J_{u_{2 i+1}}
$$

as asserted.

Next factorize $y_{i+1}$ as $b_{i+1} y_{i+1}^{\prime}$ with $b_{i+1} \in J_{R}$. This gives

$$
J \leq J_{u_{2 i+1}}=J_{u_{2 i+1} b_{i+1}} \leq J_{b_{i+1}},
$$

and once again strict inequality is impossible because of the maximality condition on $J$. Therefore $J=J_{u_{2 i+1}}$.

The dual argument now establishes that given $u_{0}, u_{1}, \ldots, u_{2 i+1} \in J(0 \leq i \leq$ $m-2$ ), then $u_{2 i+2} \in J$. Thus we have proved that $u_{0}, u_{1}, \ldots, u_{2 m-1} \in J$. Arguing as before we obtain $y_{m}=b_{m} y_{m}^{\prime}$ with $b_{m} \in J$ and $b_{m}=s_{m} u_{2 m-1} b_{m}$ for some $s_{m} \in U^{1}$. But then

$$
y_{m}=b_{m} y_{m}^{\prime}=s_{m} u_{2 m-1} b_{m} y_{m}^{\prime}=s_{m} u_{2 m-1} y_{m}=s_{m} u_{2 m} \in U^{1}
$$

which is a contradiction as $y_{m} \in S \backslash U$. This completes the proof.

I thank S. Goberstein for his suggestions concerning the exposition of this result. 


\section{REFERENCES}

1. T. E. Hall, Epimorphisms and dominions, Semigroup Forum 24 (1982), 271-283.

2. T. E. Hall and P. R. Jones, Epis are onto for finite regular semigroups, Proc. Edinburgh Math. Soc. 26 (1983), 151-162.

3. P. M. Higgins, Epis are onto for generalized inverse semigroups, Semigroup Forum 23 (1981), 255-259.

4. The commutative varieties of semigroups for which epis are onto, Proc. Edinburgh Math. Soc. 94A (1983), 1-7.

5. __ A semigroup with an epimorphically embedded subband, Bull. Austral. Math. Soc. 27 (1983), 231-242.

6. __ Epimorphisms, permutation identities and finite semigroups, Semigroup Forum 29 (1984), 87-97.

7. J. M. Howie, An introduction to semigroup theory, London Math. Soc. Monographs 7, Academic Press, New York, 1976.

8. J. M. Howie and J. R. Isbell, Epimorphisms and dominions. II, J. Algebra 6 (1967), 7-21.

9. J. R. Isbell, Epimorphisms and dominions, Proc. Conf. Categorical Algebra (La Jolla, 1965), Lange and Springer, Berlin, 1966, pp. 233-246.

10. H. E. Scheiblich and K. C. Moore, $\tau_{x}$ is absolutely closed, Semigroup Forum 6 (1973), 216-266.

11. Kunitaka Shoji, Right self-injective semigroups are absolutely closed, Mem. Fac. Sci. Shimane Univ. 14 (1980), 35-39.

12. P. G. Trotter, A non-surjective epimorphism of bands, Algebra Universalis (to appear).

Department of Mathematics, California State University, Chico, CaliforNIA 95929

Current address: Mathematics Section, Deakin University, Victoria 3217, Australia 